\title{
(6) Clinical Aspect of Dyspnea in Circulatory Disorder of the Lung
}

\author{
Tatsuya Tomomatsu and Kazuaki Sera \\ The First Department of Internal Medicine, Kobe University \\ School of Medicine
}

"Dyspnea" is not unequivocally defined, so that in the present studies of dyspnea in the circulatory disorder of the lungs, the authors have taken the view that (1) dyspnea is felt as unpleasant sensation accompanied by effort breathing, and (2) recognizable with the lowered load of exercise tolerance. Patients studied were confined to cardiopulmonary disease and particularly to mitral valvular disease and chronic obstructive pulmonary diseasè.

Attempts have been made to study (I) on correlation of the functional classification (grade of dyspnea) and the hemodynamic changes of the pulmonary circulation to the ventilatory and anoxic factors and (II) on the cardiopulmonary function at a critical level of the exercise tolerance and the effects of resultant anoxia.

The cardiac patients were classified according to the severity of dyspnea with the criteria of the New York Heart Association and the pulmonary patients by Fletscher's criteria. Some hemodynamic parameters such as mean pulmonary arterial pressure (PAm), mean pulmonary wedge pressure (PCm) and pulmonary arteriolar resistance (PAR) were taken as indices of circulatory disorder in the lung. These were all found to be in the higher level in the cardiacs group than in the pneumonic, but significant difference was seen only in PCm. The hemodynamic parameters indicated more significant correlation to the severity of dyspnea in the cardiac groups.

The pulmonary function tests carried out were expediently divided into two, namely, ventilatory and anoxic factors; the former includes the spirometrical determinations and ventilatory mechanics and the latter the diffusing capacity, gaseous distribution and blood gas.

(I) In the cardiac group, PAm has shown the correlation to a number of parameters in the ventilatory and anoxic factors and especially to the viscous work. In fact, however, there are not so significantly correlated except for the viscous work as seen in the pulmonic group. Besides, the most of the ventilatory factors were not so remarkable as those in the pulmonic. It is justifiable to consider that the mitral valvular disease characterized by the deteriorated pulmonary circulation was followed by the pulmonary disfunction. It is also understandable, therefore, that reduced diffusing capacity correlated not only to PAm, but to PCm. Speaking of the relation between the severity of dyspnea and pulmonary function in the cardiacs, the significant correlations were found in dynamic lung compliance, viscous work and pulmonary capillary blood volume. It was the viscous work to show the correlation both to 
PAm and the severity of dyspnea. In the cardiacs the factors inducing pulmonary hypertension would exert an accelerating effect on the viscous resistance of the lung, resulting in development of dyspnea.

In the pulmonic disease a number of parameters in the ventilatory and anoxic factors have shown the correlation not only to the hemodynamic factors, especially pulmonary vascular resistance, but also to the severity of dyspnea. The significant correlation was found both to the hemodynamic factor and the severity of dyspnea in FEV 1.0\%, BRR, ventilatory mechanics and blood gas. Those would be attributable to inducing the respiratory distress with the resultant deterioration of the pulmonary circulation.

It has been indicated that it is ventilatory mechanics and pulmonary capillary blood volume to be common to the cardiopulmonary diseases.

Table. 1. Correlation of Pulmonary Functions to Hemodynamic Parameters and Functional Classification in the Patients with Mitral Stenosis and Pulmonary Emphysema

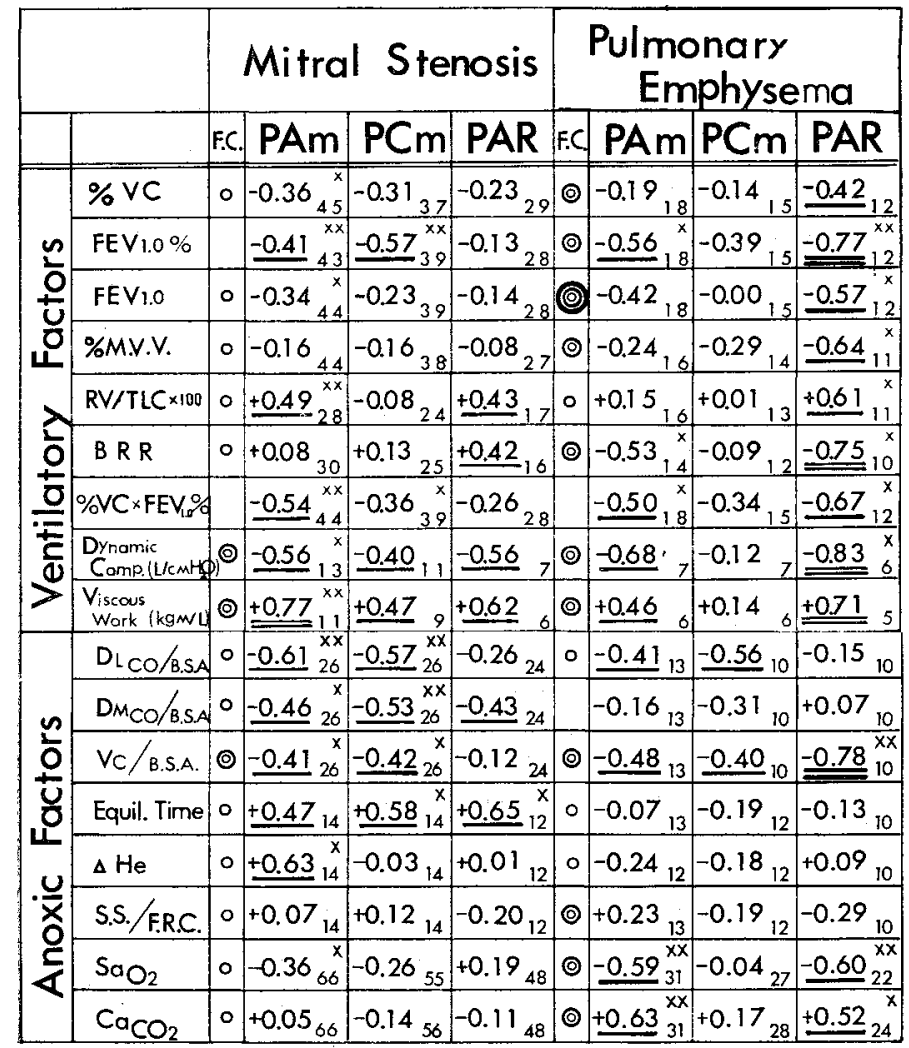

F.C.: Functional class. $\mathrm{O}, \mathrm{O}$, (9): slight, moderate and high correlation. Correlation coefficient $-: 0.4<\mathrm{r}<0.69,=: 0.7<\mathrm{r}<$ 1.0, $\times: \rho<0.05, \times \times: \rho<0.01$. 
The former involves the increased elastic and viscous resistance of the lung which required the labored respiration for fulfilling the ventilatory demand. As the pulmonary capillary blood volume has shown to have the negative correlation to ventilation/ perfusion relation obtained by determining the arterio-alveolar nitrogen difference, it is important as an anoxic factor.

(II) Using the bicycle ergometer, the exercise test was performed in the recumbent position. The load was raised until reaching the critical level. Cardiac and respiratory rate, oxygen consumption $\left(\mathrm{Vo}_{2}\right)$, carbon dioxide production $\left(\mathrm{Vco}_{2}\right)$, oxygen debt, blood gas, $\mathrm{pH}$, cardiac output and excess lactate were measured at each level of exercise. The critical level of exercise tolerance was found to be lower in the cardiopulmonic group and fell proportional to the severity of dyspnea and accompanied inevitably by the reduced maximum oxygen consumption. Repeated measurements by acetylene method during the exercise revealed the definite increase in the cardiac output in the pulmonic group and the reduced increment in the cardiacs. It is explicable from the arteriovenous oxygen difference; namely, the greater value was obtained in the latter. Beside this, the fact that the maximum oxygen consumption in cardiac group was reduced proportional to the PAm and $\mathrm{PCm}$ was considered as one of the promoting factors to the tissue anoxia inspite of no detectable anoxemia induced by loading the exercise. The pulmonary group was characterized by remarkable reduction of arterial oxygen tension with an occasional increase in arterial carbon dioxide. It is evident that the pulmonic group is in or is prone to the anoxic state.

Oxygen debt measured was increased in proportion to the load of exercise. The maximum oxygen debt in the cardiopulmonary group was of the small magnitude, showing the negative correlation to the severity of dyspnea. The normal subject is capable of augmenting the oxygen debt as much as over 10 liters on the strenuous exercise in spite of the fact that the hardly any measurable oxygen debt is found on the mild exertion with which the cardiopulmonary patients would show a sizable oxygen debt. The maximum oxygen debt which was followed by this excess lactate indicative of the limit of the anaerobic metabolism compensatory for the deficient oxygen supply is to be used as an index of exercise tolerance by which dyspnea is recognizable.

Finally, some mentions should be added to the ventilatory mechanics. which was found most closely related to dyspnea in both groups of disease. It is proved by the exercise tolerance test that dyspnea was invariably caused when the viscous work, but dynamic compliance, reached to the level of $0.09 \mathrm{~kg} . \mathrm{M} . / \mathrm{L}$.

Conclusion: Dyspnea in the mitral valvular disease and chronic obstructive pulmonary disease is caused by increase in viscous work and recognized by the reduced maximum oxygen uptake together with the maximum oxygen debt which are the indices of exercise tolerance. 